\title{
Looking for dust and molecules in Nova V4743 Sagittarii ${ }^{\star}$
}

\author{
M. Nielbock and L. Schmidtobreick \\ European Southern Observatory, Casilla 19001, Santiago 19, Chile \\ Received 8 November 2002 / Accepted 24 January 2003

\begin{abstract}
We present $1.2 \mathrm{~mm}$ continuum images and spectral line observations of $\mathrm{CO}(1-0)$ and $\mathrm{SiO}(3-2)$ rotational transitions of the recent nova V4743 Sgr. The nova is detected at $1.2 \mathrm{~mm}$ showing a variable millimetre emission. Only upper limits of $T_{\mathrm{A}}^{\star}=$ $0.06 \mathrm{~K}$ for $\mathrm{CO}$ and $T_{\mathrm{A}}^{\star}=0.03 \mathrm{~K}$ for $\mathrm{SiO}$ could be derived. We discuss the results in terms the nature of the millimetre emission favouring dust from a phase before the recent outburst as the likely radiating source. We also comment on the possibility of free-free emission from the ionised shell as the source of the measured millimetre radiation.
\end{abstract}

Key words. ISM: dust - ISM: molecules - stars: novae - stars: individual: V4743 Sgr

\section{Introduction}

V4743 Sgr was discovered as a possible nova by Katsumi Haseda (2002) at about 5 mag on September 20th, 2002. West (2002) measured its position as $\mathrm{RA}(\mathrm{J} 2000)=19^{\mathrm{h}} 01^{\mathrm{m}} 09^{\mathrm{s}} 38$, $\operatorname{Dec}(\mathrm{J} 2000)=-22^{\circ} 00^{\prime} 05^{\prime \prime} \cdot 9$ with an uncertainty of $0{ }^{\prime} \cdot 75$. Kato et al. (2002) confirmed that the object is an Fe II-class nova, and measured the $F W H M$ of the $\mathrm{H} \alpha$ emission line of $2400 \mathrm{~km} \mathrm{~s}^{-1}$. The light curves of AAVSO 2002 reveal it to be a very fast nova undergoing a steep decline from its maximum and reaching three magnitudes below maximum within 15 days. This is usually understood as the transition phase where dust formation might begin.

The formation of dust has been suggested in many novae because of a) the behaviour of their visual light curves which indicate the increase of interstellar extinction at the beginning of the transition phase, and b) the development of the infrared excess at the same time (see Bode \& Evans 1989 for an overview). The dust that forms during nova eruptions consists of a variety of mineralogies, and in some novae, simultaneous evidence is found for probably co-existing carbon-rich and oxygen-rich condensates (Evans et al. 1997).

While most recent novae have been well observed in the optical and even infrared, hardly any observation exists at sub-millimetre or millimetre wavelengths. Still, these measurements are valuable, because they allow to investigate i) the expansion of the ionised shell from emission of free-free radiation as a progenitor of the colder neutral matter, ii) the formation of molecules like $\mathrm{CO}$ and other abundant species of the interstellar medium (ISM), iii) the subsequent formation of dust, which

\footnotetext{
Send offprint requests to: M. Nielbock, e-mail: mnielboc@eso.org

* Based on observations collected with the Swedish ESO Submm Telescope at the European Southern Observatory, La Silla, Chile.
}

has a deep impact on our understanding of the enrichment of the ISM and hence the formation of stars.

The James Clerk Maxwell Telescope nova-monitoring group detected V4743 Sgr with SCUBA (Ivison 2002). On October 3rd, about 12 days after visual maximum, they measured a flux density of $39 \pm 4 \mathrm{mJy}$ at $850 \mu \mathrm{m}$ and determined an upper limit of $225 \mathrm{mJy}$ at $450 \mu \mathrm{m}$.

In this paper, we present observations of the $1.2 \mathrm{~mm}$ continuum emission and the spectral line emission of $\mathrm{CO}(1-0)$ and $\mathrm{SiO}(3-2)$ of $\mathrm{V} 4743 \mathrm{Sgr}$ taken in a range between about 20 and 60 days after the visual maximum. We compare the $1.2 \mathrm{~mm}$ emission with Ivison's 450 and $850 \mu \mathrm{m}$ data and discuss the implications for the origin of the millimetre emission.

\section{Observations and data reduction}

The observations were carried out at the SEST on La Silla, Chile. Millimetre continuum observations at $1.2 \mathrm{~mm}$ were taken using the SEST Imaging Bolometer Array (SIMBA) operating at $250 \mathrm{GHz}$ with a $F W H M$ bandwidth of $90 \mathrm{GHz}$. The maps were produced with the fast-scanning mode (Reichertz et al. 2001) without a wobbling secondary mirror. The correlated sky noise was eliminated during the data reduction process with MOPSI ${ }^{1}$. The zenith opacity at $1.2 \mathrm{~mm}$ was determined with skydips, and ranged between $\tau_{0}=0.18$ and 0.44 . Flux calibration was achieved with a series of Uranus maps. We estimate it to be accurate to $10 \%$.

Spectra of the rotational transitions of $\mathrm{CO}(1-0)$ at $115.3 \mathrm{GHz}$ and $\mathrm{SiO}(3-2)$ at $127.1 \mathrm{GHz}$ were acquired using the SESIS 100/150 heterodyne receiver providing a FWHM beam size of about $60^{\prime \prime}$. We used the acousto-optical spectrometer (AOS) at low resolution (LRS) in order to achieve the largest

\footnotetext{
${ }^{1}$ MOPSI has been developed by Dr. R. Zylka, IRAM, Grenoble, France.
} 


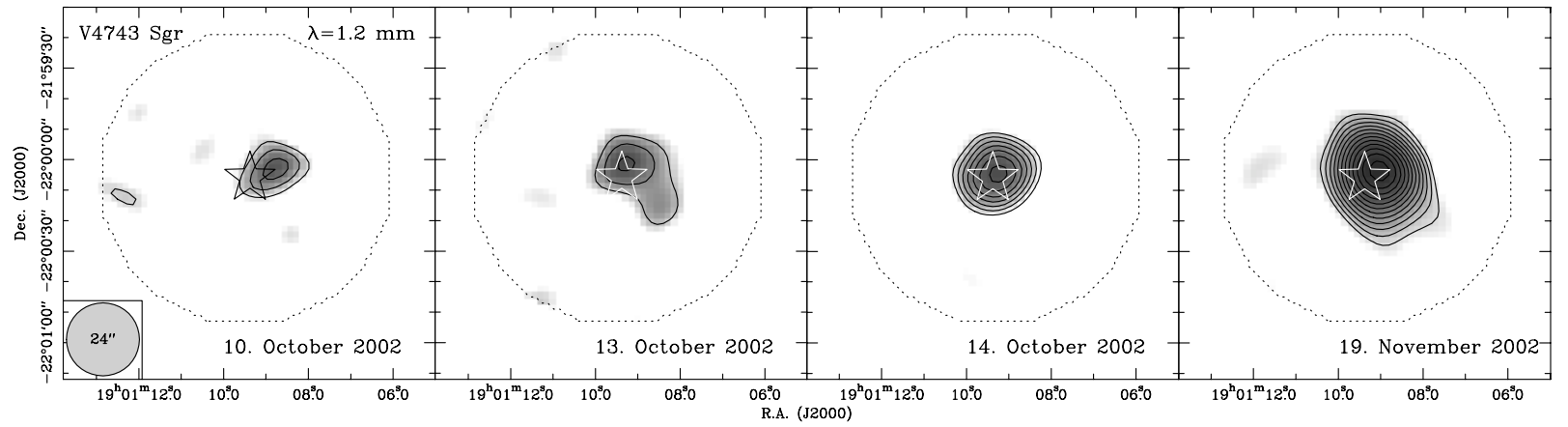

Fig. 1. The individual maps of V4743 Sgr at $1.2 \mathrm{~mm}$ continuum emission. The position of the nova as determined from optical observations is marked as a star. The beam size (lower left corner) is too large as that the structure of the source can be regarded as real.

Table 1. Details of the continuum observations with SIMBA at $\lambda=1.2 \mathrm{~mm}$ : date, number of exposures, individual integration time, and atmospheric zenith opacity.

\begin{tabular}{lllc}
\hline \hline Date (2002) & $\mathrm{No}_{\exp }$ & $t_{\exp }[\mathrm{s}]$ & $\tau_{0}$ \\
\hline 10th Oct. & 14 & 264 & $0.18-0.22$ \\
13th Oct. & 10 & 264 & $0.22-0.37$ \\
14th Oct. & 17 & 264 & $0.43-0.44$ \\
19th Nov. & 12 & 264 & $0.18-0.22$ \\
\hline
\end{tabular}

Table 2. Details of the spectral line observations: date, frequency, molecular transition, central velocity (LSR), system temperature, and integration time.

\begin{tabular}{llllll}
\hline \hline Date (2002) & $v[\mathrm{GHz}]$ & Molecule & $v_{\mathrm{LSR}}\left[\mathrm{km} \mathrm{s}^{-1}\right]$ & $T_{\text {sys }}[\mathrm{K}]$ & $t_{\text {obs }}[\mathrm{s}]$ \\
\hline 15th Oct. & 115.3 & $\mathrm{CO}(1-0)$ & 0 & 446 & 600 \\
15 th Oct. & 115.3 & $\mathrm{CO}(1-0)$ & +800 & 531 & 450 \\
15 th Oct. & 127.1 & $\mathrm{SiO}(3-2)$ & 0 & 231 & 600 \\
15 th Oct. & 127.1 & $\mathrm{SiO}(3-2)$ & +800 & 271 & 450 \\
\hline
\end{tabular}

possible velocity coverage. Two spectra for each molecule were produced, one at the centre velocity of $0 \mathrm{~km} \mathrm{~s}^{-1}$, and another with an offset of $+800 \mathrm{~km} \mathrm{~s}^{-1}$ (LSR). The separation of each two of the 1440 spectrometer channels is $0.7 \mathrm{MHz}$ being equivalent to $1.8 \mathrm{~km} \mathrm{~s}^{-1}$ and $1.6 \mathrm{~km} \mathrm{~s}^{-1}$, respectively. Dual beam switching was chosen for the rejection of the sky emission and system imbalances. A summary of the observational parameters is given in Tables 1 and 2 .

\section{Results}

V4743 Sgr was detected in all $1.2 \mathrm{~mm}$ continuum measurements with a $S / N$ of at least 4 . A confusion with another source is unlikely. Apart from rare phenomena like novae and supernovae, only quasars and similar background sources show a strong variation as witnessed in our study. 30 per $\square^{\circ}$ are expected for the detection limit of our observations (Blain et al. 1998). This gives about 0.02 sources for our map coverage.

The individual $1.2 \mathrm{~mm}$ maps are presented in Fig. 1. Note that the structures inside the source are small in comparison to the beam size (lower left corner) and are therefore probably
Table 3. Flux densities at $1.2 \mathrm{~mm}$ as determined via aperture photometry $\left(S_{\text {ap }}\right)$ within a diameter of $1^{\prime}$ and via integration over a fitted 2-dim Gaussian $\left(S_{\mathrm{G}}\right)$ with their uncertainty are listed for each date.

\begin{tabular}{lrr}
\hline \hline HJD $[2452000+]$ & $S_{\text {ap }}[\mathrm{mJy}]$ & $S_{\mathrm{G}}[\mathrm{mJy}]$ \\
\hline 558.406 & $16 \pm 4$ & $21 \pm 4$ \\
561.271 & $65 \pm 9$ & $52 \pm 9$ \\
562.342 & $30 \pm 5$ & $39 \pm 5$ \\
598.483 & $130 \pm 8$ & $142 \pm 5$ \\
\hline
\end{tabular}

not real. The flux densities have been determined in two ways: a) by integrating over a $1^{\prime}$ aperture while subtracting the background $\left(S_{\text {ap }}\right)$, and b) by fitting a two-dimensional Gaussian to the source and integrating the flux within $\left(S_{\mathrm{G}}\right)$. Table 3 lists both values for all epochs as well as the $1 \sigma$ rms noise as the photometric uncertainty. A millimetre light curve is plotted in Fig. 2. We used $S_{\mathrm{G}}$ for a further analysis. The point-like source should have a Gaussian shape, but atmospheric fluctuations distort the images of weak sources. Therefore, a simple aperture photometry might be misleading. In addition, the integration over a Gaussian is more comparable to on-off measurements.

The spectral line observations of the $\mathrm{CO}(1-0)$ and the $\mathrm{SiO}(3-2)$ rotational transitions only provided upper limits of $T_{\mathrm{A}}^{\star}=0.06 \mathrm{~K}$ for the $\mathrm{CO}$ and $T_{\mathrm{A}}^{\star}=0.03 \mathrm{~K}$ for the $\mathrm{SiO}$ measurements within the covered velocity range.

\section{Discussion}

Millimetre continuum radiation from novae can arise from thermal dust emission (e.g. Evans et al. 1997) or as free-free emission from hot ionised gas (e.g. Ivison et al. 1993). The decision on which is the dominant emission source is made on the shape of the spectral energy distribution (SED), i.e. the slope $\alpha$ of its Rayleigh-Jeans tail. The SED of dust emission is usually described by a modified blackbody law. The flux density $S_{v}$ at a frequency $v$ is given by

$S_{v}=\Omega B_{v}(T)\left(1-\exp \left(-\tau_{v}\right)\right), \quad \tau_{v}=\left(v / \nu_{\mathrm{c}}\right)^{\beta}$

where $\Omega$ is the solid angle of the emitting source, $T$ the dust temperature, $\tau_{v}$ the optical depth, and $B_{v}(T)$ the Planck function. The optical depth is a function of the frequency with an exponent $\beta=\alpha-2$, which often is referred to as the submm 
emissivity index, and $v_{\mathrm{c}}$ is the critical frequency, i.e. the frequency where $\tau_{v}=1$. Values between $\beta=1$ and $\beta=2.5$ are found for different dust grains. Free-free emission instead yields a flat $\operatorname{SED}(\alpha=-0.1)$ for optically thin and $\alpha=2$ for optically thick media (e.g. Chen et al. 1995).

Unfortunately, no simultaneous measurements at $\mathrm{mm}$ and submm exist for V4743 Sgr. Therefore, we calculated $1.2 \mathrm{~mm}$ values for the time of the SCUBA measurements for the cases of free-free and dust emission, and included them in Fig. 2. The steepest submm slope that is consistent with the SCUBA data is $\alpha=2.6$. If the mm emission were due to free-free radiation from an expanding ionised shell, it would be difficult to explain the decreasing flux density in the beginning. Hence, free-free emission seems to be an unlikely source, and we favour the dust interpretation. Additional evidence comes from the optical light-curves provided by AAVSO. Coincidently with the increase in the submm, they show a rise of the $R$-band intensity, while values at shorter wavelengths remain constant.

The formation of dust in novae is usually observed by an increasing IR emission accompanied by a prominent dip in the visual light curve of the nova which can be explained by extinction introduced by newly formed dust. Such an extinction dip has not been observed for V4743 Sgr. Together with the lack of any emission from $\mathrm{CO}$ and $\mathrm{SiO}$ molecules, we conclude that the millimetre emission does not originate from newly formed but old dust. It might be either a remnant from the pre-CV phase or a former nova outburst. In that case, V4743 Sgr would actually be a recurrent nova. A similar interpretation was made for V4444 Sgr which showed a rise in the IR without any dip in the optical light curve (Venturini et al. 2002). If we follow the argument of Kawabata et al. (2000), we can use Eq. (6) of Clayton $\&$ Wickramasinghe (1976) to verify that dust cannot be present inside a radius of $\sim 50 \mathrm{AU}$ from the nova. The largest possible radius of the expanding nova shell of V4743 Sgr can be derived by extrapolating the expansion speed measured from the $\mathrm{H} \alpha$ line width of $2400 \mathrm{~km} \mathrm{~s}^{-1}$ for 23 days to the first millimetre maximum. This yields $4.8 \times 10^{9} \mathrm{~km}$ or $32 \mathrm{AU}$. Hence, the dust cannot originate from the recent outburst. The presence of preexisting dust around novae has been shown for several more cases (e.g. Malakpur 1977).

$M_{\text {dust }}=\frac{S_{v} D^{2}}{\kappa_{v} B_{v}(T)}$.

Equation (2) allows to estimate the mass of the surrounding dust, if $D$ the distance to the source, $\kappa_{v}$ the mass absorption coefficient at the measured frequency $v$, and $T$ the dust temperature are known. $\kappa_{v}$ can differ at least by a factor of 10 , depending on the local environment. In our case, we adopt a value of $\kappa_{250}=2 \mathrm{~cm}^{2} \mathrm{~g}^{-1}$, which is typical for circumstellar dust (Krügel \& Siebenmorgen 1994) consisting of relatively small grains (Kawabata et al. 2000).

The determination of the distance $D$ of CVs is generally quite difficult, although some methods exist to estimate the distance modulus of novae. Firstly, the absolute visual magnitude of a nova 15 days after the maximum is supposed to be similar for all novae, i.e. $M_{V}(15)=-5.69 \pm 0.14 \mathrm{mag}$ (Capaccioli et al. 1990, and others herein). Secondly, the absolute visual magnitude of a nova at its maximum is related to the time $t_{2}$,

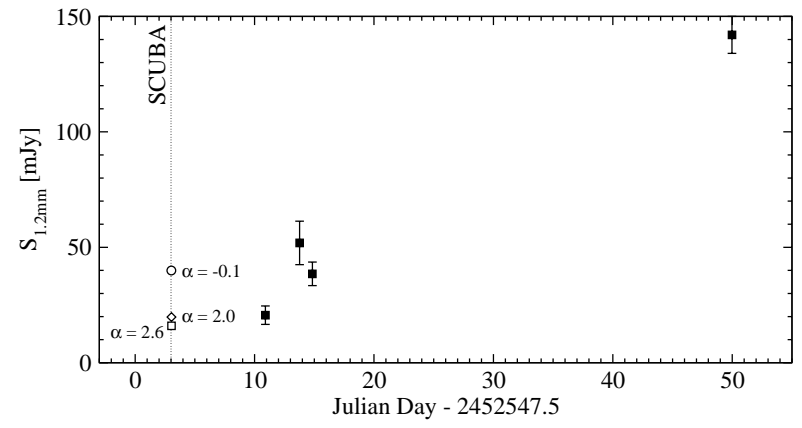

Fig. 2. Millimetre light curve of V4743 Sgr. The four rightmost squares mark the observed $1.2 \mathrm{~mm}$ flux densities $S_{\mathrm{G}}$. The intensities at the date of the SCUBA measurement (indicated as a vertical dotted line) have been extrapolated from the SCUBA data at $850 \mu \mathrm{m}$ for different submm slopes $\alpha$.

in which the nova descends by 2 mag (Cohen 1988). We estimated the distance modulus of V4743 Sgr with both methods and get $(m-M)=13.9 \pm 0.4$ which yields a maximum possible distance of $6 \mathrm{kpc}$. However, the crucial point is to measure the interstellar extinction towards the nova. The region looks very patchy and HD 176537, the closest star with a measured extinction, still lies $40^{\prime}$ away from the nova itself and hence its value $A_{V}=1.42 \mathrm{mag}$ (Guarinos 1992) might differ quite a lot from that of V4743 Sgr. Additionally, its heliocentric distance is only about $100 \mathrm{pc}$. Assuming that HD 176537 and the nova are sharing the same interstellar environment, and scaling the extinction value accordingly, we estimate the distance of V4743 Sgr to be around $D=400 \mathrm{pc}$ with $A_{V}=5.7 \mathrm{mag}$. Using the $J-H$ and $H-K$ colours of the 2Mass point sources in a vicinity of $1^{\prime}$ in comparison with intrinsic colours from Bessel \& Brett (1988), we estimate the average extinction as $A_{V}=3.4 \pm 0.5$ mag which yields $D=1200$ pc for the nova. We want to point out that these values are very speculative and have to be regarded with caution. For this reason, we relate the mass estimates to a standard distance of $100 \mathrm{pc}$.

A well established estimate of the dust temperature is crucial for deriving a proper dust mass from millimetre observations. The present data are insufficient to achieve this, and temperature determinations are sparse throughout the literature. Evans et al. (1997) quote a dust temperature of some $750 \mathrm{~K}$ for V705 Cas after 250-300 days after the nova eruption, and Bode \& Evans (1989) give values between 700 and $800 \mathrm{~K}$ for NQ Vul between 80 and 240 days after the outburst. However, these values have been derived from infrared measurements during the ionisation phase and therefore do not trace the coolest dust involved. Consequently, they may not be appropriate for our purposes.

On the other hand, Lynch et al. (2001) derive a temperature of $280 \mathrm{~K}$ for the cool dust component in V445 Pup. Beck et al. (1990) present models of nova environments, in which one of the most important ingredients for dust formation, $\mathrm{C}_{\mathrm{I}}$, attains a temperature of about $100 \mathrm{~K}$ already soon after the nova eruption. We therefore use typical dust temperatures of 100 and $300 \mathrm{~K}$ for the mass determination. The so derived dust masses are listed in Table 4 for 100 and $300 \mathrm{~K}$, respectively. As discussed above, they are scaled to a distance of $100 \mathrm{pc}$. 
Table 4. Dust masses and column densities as calculated from Eq. (2). We assume a standard distance of $100 \mathrm{pc}$ for estimating the mass.

\begin{tabular}{ccccc}
\hline \hline HJD & \multicolumn{2}{c}{$M_{\text {dust }}\left(\frac{D}{100 \mathrm{pc}}\right)^{-2}\left[M_{\odot}\right]$} & $N\left(\frac{D}{100 \mathrm{pc}}\right)^{-2}\left[\mathrm{~cm}^{-2}\right]$ \\
{$[2452000+]$} & $T=100 \mathrm{~K}$ & $T=300 \mathrm{~K}$ & $T=100 \mathrm{~K}$ & $T=300 \mathrm{~K}$ \\
\hline 558.406 & $2.8 \times 10^{-6}$ & $8.9 \times 10^{-7}$ & $7.0 \times 10^{20}$ & $2.2 \times 10^{20}$ \\
561.271 & $6.9 \times 10^{-6}$ & $2.2 \times 10^{-6}$ & $1.7 \times 10^{21}$ & $5.5 \times 10^{20}$ \\
562.342 & $5.2 \times 10^{-6}$ & $1.7 \times 10^{-6}$ & $1.3 \times 10^{21}$ & $4.2 \times 10^{20}$ \\
\hline
\end{tabular}

We determined column densities of the surrounding dust from the derived masses. The projected area in the line of sight was calculated from the lowest possible dust radius, i.e. $50 \mathrm{AU}$ (see above). Since the area can be larger than this, these column densities are only an upper limit and are also listed in Table 4.

If we assume the distance estimates from above, we get average dust masses of about $5 \times 10^{-5} M_{\odot}$ for $D=400 \mathrm{pc}$ and $5 \times 10^{-4} M_{\odot}$ for $D=1200 \mathrm{pc}$. The referring column densities are $1 \times 10^{22} \mathrm{~cm}^{-2}$ and $1 \times 10^{23} \mathrm{~cm}^{-2}$, respectively. We recall that a distance of $400 \mathrm{pc}$ is probably a gross underestimate. We therefore think that the actual values are more likely to be found at the higher end.

If the flux variation were due to forming dust, the dust formation rate $\dot{M}_{\text {dust }}$ could be estimated from the dust masses between the first two measurements ( 2.9 days). For the distance estimates from above we find $3.5 \times 10^{23}$ and $3.1 \times 10^{24} \mathrm{~g} \mathrm{~s}^{-1}$, respectively. We compare these dust formation rates with the results from two other novae, Nova Serpentis 1978 (LW Ser) and Nova Cassiopeiae 1993 (V705 Cas). Gehrz et al. (1980) measured the mass of the dust produced during the outburst of LW Ser from infrared observations. The infrared data show a steep rise to a maximum within 41 days, which we take as the time of the dust formation. The average dust formation rate is therefore $1.8 \times 10^{20} \mathrm{~g} \mathrm{~s}^{-1}$. Evans et al. $(1996,1997)$ show a visual light curve of V705 Cas with a prominent extinction dip of 29 days, which we define as the dust formation phase. The average dust formation rate in this case yields $7.9 \times 10^{18} \mathrm{~g} \mathrm{~s}^{-1}$. Our rates, if real, would be at least 2000 times higher. A factor of about 17000 , however, seems more likely. We interpret this discrepancy again as an indication that the radiating dust was most likely not produced after the nova eruption.

$\tau_{v}=N m_{\mathrm{d}} \kappa_{v}$.

From Eq. (3) we determine an average optical depth of the medium at $1.2 \mathrm{~mm}$ of $\left\langle\tau_{250}\right\rangle=9 \times 10^{-3}$, where $N$ is the column density and $m_{\mathrm{d}}=4.5 \times 10^{-26} \mathrm{~g}$ is the mass of a dust particle. This is taken as $1 \%$ of the particle mass of the ISM, where we correct for the typical mass abundance of helium. With Eq. (1) we can calculate the frequency for which the medium becomes optically thick. Transforming this frequency into a wavelength we get $\sim 175 \mu \mathrm{m}$ for $\beta=0.6$. The size of the bulk of the involved scattering dust particles should be of the order of $a=\lambda / 2 \pi$, i.e. $38 \mu \mathrm{m}$.

\section{Summary}

V4743 Sgr was detected at $1.2 \mathrm{~mm}$, the flux density varying strongly in time. A local intensity maximum has been found around day 23 after the visual maximum. It continued to rise steeply afterwards. The spectral line observations yielded only upper limits for $\mathrm{CO}(1-0)$ and $\mathrm{SiO}(3-2)$. The dominant emission source is found to be heated dust rather than free-free emission. We have roughly estimated the distance of the nova as $1200 \pm 300 \mathrm{pc}$. Using this value, the dust mass at the first maximum ranges between $3 \times 10^{-4} M_{\odot}$ and $1 \times 10^{-3} M_{\odot}$ depending on its temperature. The high dust mass, the accordingly high formation rate, the absence of molecules, the lack of any dip in the visual light curve while the $R$-band magnitude rises simultaneously with the mm flux, and the small shell radius favour the idea that the observed dust has already been present in the system as a remnant of either the pre-CV common envelope phase or a former nova outburst. Dust grains were estimated to have sizes around $38 \mu \mathrm{m}$.

Acknowledgements. In this research, we have used, and acknowledge with thanks, data from the AAVSO International Database, based on observations submitted to the AAVSO by variable star observers worldwide. This publication makes furthermore use of data products from the Two Micron All Sky Survey, which is a joint project of the University of Massachusetts and the Infrared Processing and Analysis Center/California Institute of Technology, funded by the National Aeronautics and Space Administration and the National Science Foundation. We also thank Andreas Lundgren and Dr. Lars-Åke Nyman for carrying out the November observation.

\section{References}

Beck, H., Gail, H.-P., \& Sedlmayr, E. 1990, A\&A, 238, 283

Bessel, M. S., \& Brett, J. M. 1988, PASP, 100, 1134

Blain, A. W., Ivison, R. J., \& Smail, I. 1998, MNRAS, 296, L29

Bode, M. F., \& Evans, A. 1989, in Classical Novae, ed. M. F. Bode \& A. Evans, 163

Capaccioli, M., Della Valle, M., D’Onofrio, M., \& Rosino, L. 1990 ApJ, 360, 63

Chen, H., Zhao, J. H., \& Ohashi, N. 1995, ApJ, 450, L71

Clayton, D. D., \& Wickramasinghe, N. C. 1976, Ap\&SS, 42, 463

Cohen, J. G. 1988, The Extragalactic Distance Scale, ASP Conf. Ser, 4,114

Evans, A., Geballe, T. R., Rawlings, J. M. C., \& Scott, A. D. 1996, MNRAS, 282, 1049

Evans, A., Geballe, T. R., Rawlings, J. M. C., Eyres, S. P. S., \& Davies, J. K. 1997, MNRAS, 292, 192

Gehrz, R. D., Grasdalen, G. L., Hackwell, J. A., \& Ney, E. P. 1980, ApJ, 237, 855

Guarinos, J. 1992, in VizieR On-line Data Catalog: V/86

Haseda, K. 2002, IAU Circ., 7975

Ivison, R. J., Hughes, D. H., Lloyd, H. M., Bang, M. K., \& Bode, M. F. 1993, MNRAS, 263, L43

Ivison, R. J. 2002, IAU Circ., 7988

Kato, T., Fujii, M., \& Ayani, K. 2002, IAU Circ., 7975

Kawabata, K. S., Hirata, R., Ikeda, Y., et al. 2000, ApJ, 540, 429

Krügel, E., \& Siebenmorgen, R. 1994, A\&A, 288, 929

Lynch, D. K., Russell, R. W., \& Sitko, M. L. 2001, AJ, 122, 3312

Malakpur, I. 1977, Ap\&SS, 47, 49

Reichertz, L. A., Weferling, B., Esch, W., \& Kreysa, E. 2001, A\&A, 379,735

Venturini, C. C., Rudy, R. J., Lynch, D. K., Mazuk, S., \& Puetter, R. C. 2002, AJ, 124, 3009

West, D. 2002, IAU Circ., 7975 